\title{
Immunotherapy-Resistant Vitreoretinal Metastatic Melanoma
}

\author{
Arthi Venkat Elaine M. Binkley Sunil Srivastava Naveen Karthik \\ Arun D. Singh
}

Cole Eye Institute, Cleveland Clinic, Cleveland, $\mathrm{OH}$, USA

\author{
Keywords \\ Immunotherapy · Vitreoretinal metastasis · Vitreoretinal \\ diseases and surgery
}

\begin{abstract}
Purpose: To describe 2 cases of vitreoretinal metastases in patients treated with immunotherapy for metastatic melanoma. Methods: Retrospective case series. Results: We present 2 patients with metastatic melanoma treated with systemic immunotherapy with subsequent development of ocular vitreoretinal metastasis. The first patient was a male with metastatic melanoma from a site of unknown origin that was in complete remission following a course of ipilimumab and nivolumab therapy. He presented to an outside provider for evaluation of vitritis and a pigmented lesion in the right eye that was presumed secondary to toxoplasmosis. After failing initial management with oral antibiotics, he underwent diagnostic pars plana vitrectomy, and vitreous biopsy was consistent with metastatic melanoma to the vitreous. He was additionally found to have an elevated pigmented retinal mass consistent with a retinal metastasis from melanoma that initially failed treatment with plaque brachytherapy and ultimately required enucleation. The second case was a monocular male with metastatic melanoma from cutaneous melanoma with decreased vision 3 months after the initiation of nivolumab therapy. He presented with dense vitreous debris in his seeing eye and was thought to have nivolumabassociated inflammation. He was initially treated with diflu-
\end{abstract}

prednate with improved vision and decrease in vitreous debris, but developed dense pigment deposition in the affected eye later in the treatment course. Diagnostic pars plana vitrectomy was performed, and vitreous biopsy was positive for malignant melanoma cells. His systemic disease was in remission at the time of diagnosis of ocular metastasis. External beam radiation was recommended given his monocular status. Conclusion: Vitreoretinal metastasis can develop despite favorable systemic response to immunotherapy in patients with metastatic cutaneous melanoma. Lack of ocular penetration and extension of life span with immunotherapeutic agents may be the underlying mechanism for vitreoretinal metastasis.

c) 2020 S. Karger AG, Basel

\section{Introduction}

In contrast to choroidal metastasis, the most common site of uveal metastasis from systemic malignancy, retinal and vitreous metastasis is a rare occurrence $[1,2]$. Vitreous and retinal metastases have been reported in patients with wide-spread metastasis from lung, breast, esophageal, gastric, skin and colon cancer, [2-8]. Of the reported cases of vitreoretinal metastases, many have occurred in the setting of metastatic cutaneous melanoma [2, 4, 7-12].

The advent of immunotherapy for the treatment of metastatic melanoma has increased survival for patients with previously limited options [13]. In particular, check-

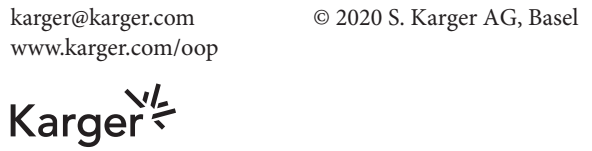


point inhibitors have gained favor in the treatment of metastatic melanoma and other cancers. However, this class of medications is known to cause inflammatory adverse events, among them uveitis of varying severity [14]. Vitreous metastasis can masquerade as inflammation, making the diagnosis challenging in the setting of checkpoint inhibitor therapy. The diagnosis of retinal lesions can be complicated by an overlying vitritis, and the lesions are often unusual appearing and can appear as perivascular, pigmented lesions invading the retina $[2,4,8,9$, 11]. Additionally, the ocular penetration of these agents and how they might affect the incidence of vitreal and retinal metastasis is unknown.

We present 2 cases of vitreoretinal metastasis of melanoma that developed despite complete response of systemic metastatic disease to checkpoint inhibitor therapy.

\section{Case Report}

Case 1

A 66-year-old man diagnosed with metastatic melanoma of unknown site of origin and involvement of the throat, lungs, and liver presented in complete remission on ipilimumab and nivolumab. Eight months following completion of immunotherapy, he developed new floaters in the right eye and found to have vitritis and a pigmented chorioretinal scar in the inferotemporal periphery of the right eye presumed to be toxoplasmosis. Pars plana vitrectomy with vitreous biopsy identified cells consistent with metastatic melanoma. The previously noted pigmented chorioretinal scar was now noted to be elevated, concerning for retinal metastasis, and he was referred for further evaluation and management of the lesion. Systemic imaging (MRI and CT scans) was negative for metastatic disease elsewhere.

Visual acuity was 20/30 in both eyes. His intraocular pressure was elevated to $33 \mathrm{~mm} \mathrm{Hg}$ in the right eye and was normal at 19 $\mathrm{mm} \mathrm{Hg}$ in the left eye. The angle was open and without neovascularization on gonioscopy of the right eye. Bilateral poliosis of the eyelashes and eyebrows were noted which reportedly occurred during immunotherapy. On dilated funduscopic exam, there was $2+$ anterior vitreous cell in the anterior vitreous skirt of the right eye. There was an $8.5 \times 7.0 \times 1.9 \mathrm{~mm}$ heavily pigmented retinal lesion centered at 7:00 near the equator with overlying intraretinal hemorrhage and a depigmented anterior border (Fig. 1a). B-scan echography showed a dome-shaped, medium-to-highly reflective lesion (Fig. 1b). Fluorescein angiography showed blockage in the areas of pigmentation and intraretinal hemorrhage without leakage (Fig. 1c). Optical coherence tomography demonstrated that the lesion was intraretinal (Fig. 1d). These clinical and imaging findings were consistent with retinal metastasis, and a discussion was held with the patient and his medical oncologist. Given the absence of metastatic disease elsewhere and the peripheral location of the lesion with good visual prognosis, the patient elected to proceed with episcleral plaque brachytherapy to the lesion in his right eye. However, approximately 1 week following plaque removal, he demonstrated an increase in vitreous cell and multiple new retinal lesions adjacent to the original lesion. At this point, enucleation of the right eye was performed. Pathology demonstrated treated uveal melanoma of the epithelioid type, without remaining identifiable choroidal mass post-treatment, but with diffuse involvement of the globe including ciliary body, trabecular meshwork, vitreous, anterior chamber, and retina. The largest retinal focus measured 9 $\mathrm{mm} \times 0.4 \mathrm{~mm}$. All margins including optic nerve were negative for neoplasm.
Fig. 1. Images for case 1. a Fundus photo demonstrating pigmented retinal lesion. b B-scan echography demonstrating a dome-shaped retinal lesion with medium/ high internal reflectivity. c Fluorescein angiography demonstrating blockage from pigment and hemorrhage in the area of the lesion. d Optical coherence tomography demonstrating intraretinal location of the lesion.
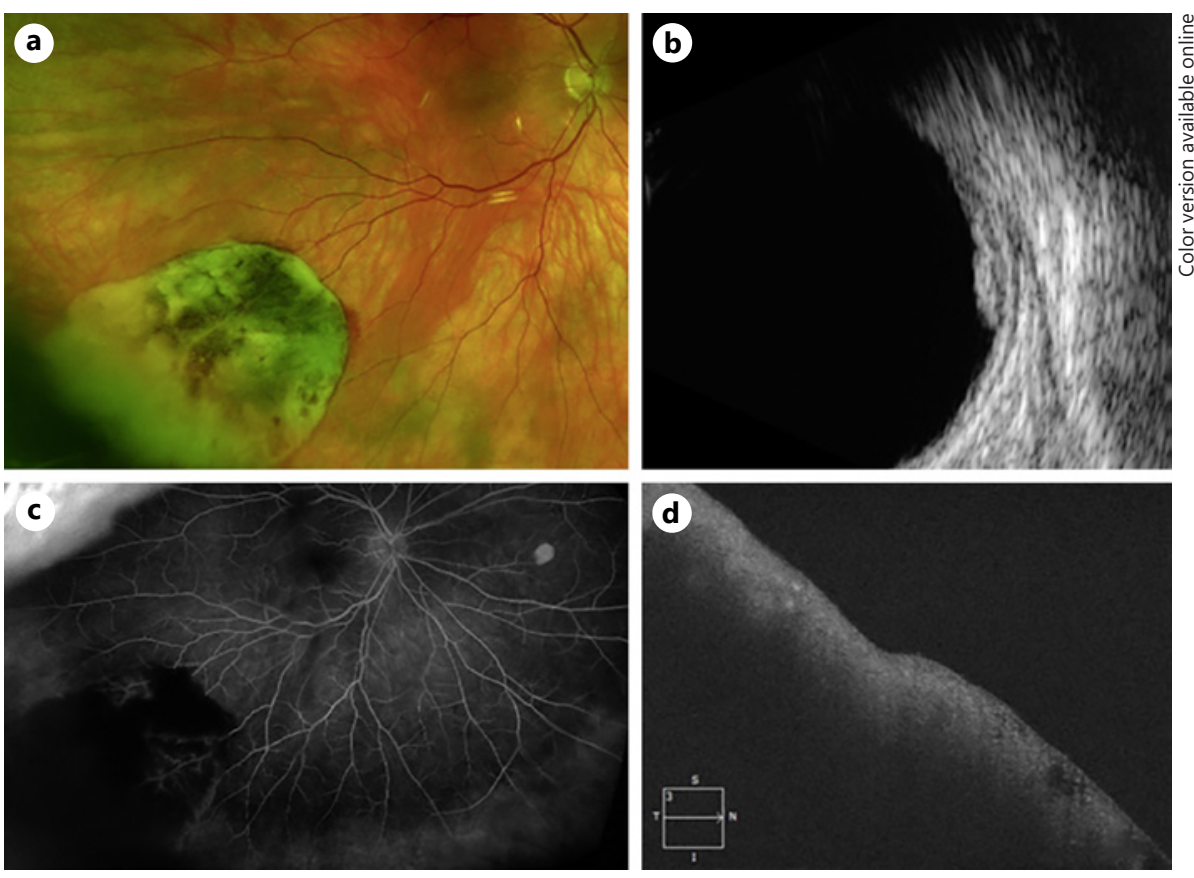

Vitreoretinal Metastasis and Immunotherapy
Ocul Oncol Pathol 2021;7:62-65 DOI: $10.1159 / 000511187$ 


\section{Case 2}

An 80-year-old monocular male with metastatic cutaneous melanoma was started on a palliative course of nivolumab. Following the third cycle, he experienced onset of blurry vision. Visual acuity on presentation was 20/60 in the right eye, and light perception in the left eye due a history of trauma. Initial examination showed dense vitreous debris in the right eye with 1-2+ vitreous cell; no vitritis was noted in the fellow eye (Fig. $2 \mathrm{a}$ ).

Fluorescein angiography and indocyanine green demonstrated significant blockage from vitreous debris in the right eye (Fig. 2b) and were unremarkable in the left eye. Based on the timeline of the patient's symptoms, the highest suspicion was for secondary uveitis from the nivolumab. Patient was started on difluprednate $0.05 \%$ eyedrops hourly in the right eye.

After 1 week, the vision (20/40) and symptoms were markedly improved on difluprednate.

Examination revealed improved vitreous debris in the right eye. Nivolumab therapy was continued concurrently with local therapy under close monitoring. Patient reported stable vision on follow-up after 4 weeks. However, examination of the right eye showed diffuse pigmented debris in the vitreous and pigment deposition on the anterior and posterior lens capsule (Fig. $2 \mathrm{c}, \mathrm{d}$ ). The sudden change in appearance was concerning for metastatic melanoma. B-scan showed vitreous debris without mass lesions, and no masses were observed on UBM. Given stable systemic disease and vision in the setting of monocular status, the decision was made to monitor the patient closely on topical therapy only. However, his vision dropped to count fingers within several weeks. Diagnostic pars plana vitrectomy was performed, and cytology was positive for melanoma cells (Fig. 3).

The patient had re-staging CT and PET scans that demonstrated stability from the standpoint of his systemic disease following nivolum$\mathrm{ab}$ therapy. Options were discussed including enucleation versus external beam radiation; palliative radiotherapy was deemed the best option, and he received $30 \mathrm{~Gy}$ in 10 fractions. However, in spite of radiotherapy, he continued to have significant pigmented debris covering his intraocular lens (IOL) limiting his visual acuity. One day following a second vitrectomy to clear debris from his IOL, he fell at home and died from a large intracranial bleed secondary to head trauma.

\section{Discussion and Conclusion}

Vitreoretinal metastasis from cutaneous melanoma is well known [4, 7-9]. However, there has been a recent increase in published cases of vitreoretinal metastasis in patients whose systemic metastatic melanoma is wellcontrolled on checkpoint inhibitor therapy [10-12]. In one reported case of vitreoretinal metastasis with responsive systemic disease on a PD-1 inhibitor, the patient developed delayed intracranial involvement and ul-
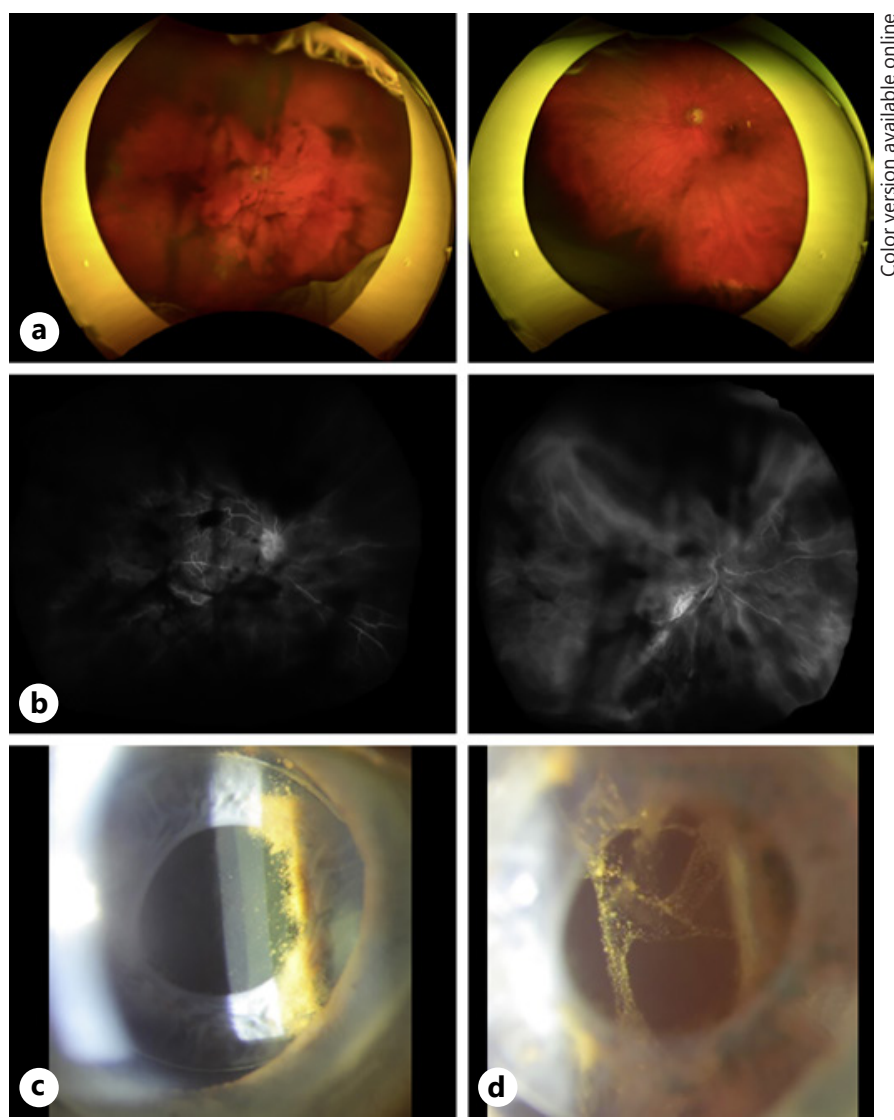

Fig. 2. Images for case 2. a Fundus photos of both eyes demonstrating dense vitreous debris in the right eye. $\mathbf{b}$ Fluorescein angiography and indocyanine green angiography demonstrating blockage from dense vitreous debris in the right eye. c, d Slit lamp photos demonstrating dense pigment deposition on the intraocular lens and in the vitreous following topical steroid therapy.
Fig. 3. Image for case 2. Pathology slide demonstrating malignant melanoma cells from vitreous sample.
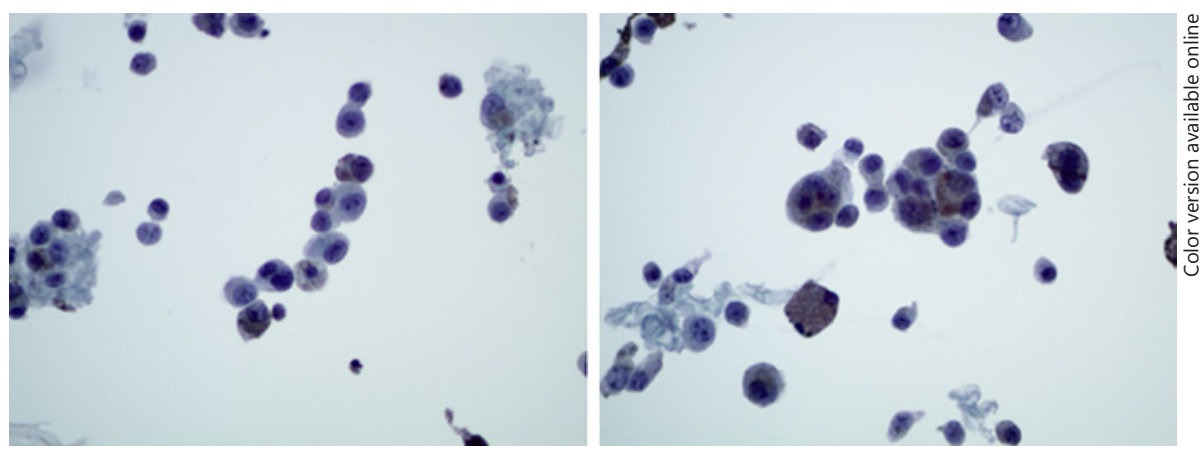
timately passed away [11]. In contrast to these cases, our second case presented with isolated vitreous metastasis without underlying retinal or choroidal involvement. This presentation is particularly rare and can easily masquerade as secondary uveitis from checkpoint inhibitor therapy. The vitreous cells seen in metastasis have been described as "tan and globular," and this appearance should therefore raise suspicion for metastatic disease over uveitis.

The cause of vitreoretinal metastasis in the setting of systemically effective immunotherapy remains unclear. It is possible that patients, who previously did not survive long enough to develop intraocular metastasis, are simply surviving longer on immunotherapy. Additionally, immunotherapy may be unable to penetrate the eye in some patients, perhaps because of its status as an immune privileged site.

As survival with metastatic melanoma treated with immunotherapy agents improves, it is important for ophthalmologists to be aware of ocular metastasis. Management of ocular metastasis should involve a careful discussion with the patient and their treating oncologist. It is additionally reasonable to review neuro-imaging with diagnosis of vitreoretinal metastasis to rule out CNS metastasis.

\section{Statement of Ethics}

This is an IRB-approved study (IRB \#13-788 - Medical Records and Imaging in Uveitis Patients). The consent signed by the patients under the IRB allowed for the use of medical records and images. Both patients whose cases are described in this publication are now deceased.

\section{Conflict of Interest Statement}

The authors have no conflicts of interest to declare.

\section{Funding Sources}

Unrestricted Grant Award from Research to Prevent Blindness to the Department of Ophthalmology, Cole Eye Institute (RPB1508DM)

\section{Author Contributions}

Arthi Venkat: conception/design of work, data collection, manuscript drafting. Elaine M. Binkley: conception/design of work, data collection, manuscript drafting. Sunil Srivastava: conception/ design of work, data collection, final approval for publication. Naveen Karthik: data collection, manuscript drafting. Arun D. Singh: conception/design of work, data collection, final approval for publication.

\section{References}

1 Shields CL, Shields JA, Gross NE, Schwartz GP, Lally SE. Survey of 520 eyes with uveal metastases. Ophthalmology. 1997 Aug; 104(8):1265-76.

2 Shields CL, McMahon JF, Atalay HT, Hasanreisoglu M, Shields JA. Retinal metastasis from systemic cancer in 8 cases. JAMA Ophthalmol. 2014 Nov;132(11):1303-8.

3 Apte RS, Dibernardo C, Pearlman JR, Patel S, Schachat AP, Green WR, et al. Retinal metastasis presenting as a retinal hemorrhage in a patient with adenocarcinoma of the cecum. Arch Ophthalmol. 2005 Jun;123(6):850-3.

4 Letson AD, Davidorf FH. Bilateral retinal metastases from cutaneous malignant melanoma. Arch Ophthalmol. 1982 Apr;100(4):6057.

5 Kim CY, Ha CW, Lee SC. Vitreous and retinal metastasis from gastric cancer. Eur J Ophthalmol. 2010 May-Jun;20(3):615-7.

6 Mack HG, Jakobiec FA. Isolated metastases to the retina or optic nerve. Int Ophthalmol Clin. 1997;37(4):251-60.
7 Krema H, Fernandes B, Simpson R, Lutchman C, Yucel Y. Diffuse metastatic melanoma to the retina: a clinicopathologic report. Can J Ophthalmol. 2013;48(2):e20-e21. https:// doi.org/10.1016/j.jcjo.2012.10.006

8 Rosenberg C, Finger PT. Cutaneous malignant melanoma metastatic to the eye, lids, and orbit. Surv Ophthalmol. 2008 May-Jun;53(3): 187-202.

9 Breazzano MP, Barker-Griffith AE. Features of Cutaneous Malignant Melanoma Metastatic to the Retina and Vitreous. Ocul Oncol Pathol. 2015 Dec;2(2):80-5.

10 Kanavati S, Ottensmeier C, Foria V, Krishnan R. Bilateral Metastatic Cutaneous Melanoma To Retina and Vitreous After Ipilimumab Treated With Pars Plana Vitrectomy and Radiotherapy. Retin Cases Brief Rep. 2018;12(3): 184-7.
11 Manusow JS, Khoja L, Pesin N, Joshua AM, Mandelcorn ED. Retinal vasculitis and ocular vitreous metastasis following complete response to $\mathrm{PD}-1$ inhibition in a patient with metastatic cutaneous melanoma. J Immunother Cancer. 2014 Dec;2(1):41.

12 Sia DI, Thaung C, O'Hanlon-Brown C, Cohen VM, Sagoo MS. Immune privilege: failure of immunotherapy in controlling metastatic cutaneous melanoma to the eye. Melanoma Res. 2018 Aug;28(4):359-62.

13 Franklin C, Livingstone E, Roesch A, Schilling $B$, Schadendorf D. Immunotherapy in melanoma: recent advances and future directions. Eur J Surg Oncol. 2017 Mar;43(3):604-11.

14 Dalvin LA, Shields CL, Orloff M, Sato T, Shields JA. Checkpoint Inhibitor Immune Therapy: Systemic Indications and Ophthalmic Side Effects. Retina. 2018 Jun;38(6): 1063-78. 